\title{
Pentoxifylline-Tocopherol and A-PRF Associated with Sequestrectomy for the Treatment of Severe Mandibular Osteoradionecrosis: Case Report and Long-Term Follow-Up
}

\section{Salvador Valladares-Pérez ${ }^{1,2 *}$, Osvaldo Gahona-Gutiérrez ${ }^{1,3}$, Pablo Romero-Araya ${ }^{4}$ and Juan Pablo Vargas-Buratovic ${ }^{2}$}

\author{
${ }^{1}$ Servicio de Cirugía Maxilofacial, Hospital El Carmen Dr. Luis Valentín Ferrada, Maipú, Santiago- Chile \\ ${ }^{2}$ Escuela de Odontología, Facultad de Medicina, Pontificia Universidad Católica de Chile, Santiago- Chile \\ ${ }^{3}$ Facultad de Odontología, Universidad Finis Terrae, Santiago-Chile \\ ${ }^{4}$ Escuela de Odontología, Facultad de Medicina, Universidad Austral de Chile, Valdivia-Chile
}

*Corresponding author: Salvador Valladares Pérez, Hospital El Carmen Dr. Luis Valentín Ferrada, Santiago, Camino A Rinconada 1201 Maipú, Santiago-Chile, Tel: 569-82944382

\begin{abstract}
Osteoradionecrosis treatment is challenging for clinicians. Throughout history, the study of osteoradionecrosis and its pathophysiological understanding has given the guidelines for managing this pathology. Currently, osteoradionecrosis is understood as a radiation-induced fibro-degenerative process, so its management focuses on reducing the molecular mediators involved during the process. We present the case of a 61-year-old male patient with multiple comorbidities and medical history of the floor of mouth cancer treated with radiotherapy. After extracting a mandibular tooth, osteoradionecrosis with pathologic fracture and orocutaneous fistula developed in the mandibular body. Due to multiple advanced inferior extremities atheromatosis and comorbidities, a fibula microvascular free flap was contraindicated.

Thereby, long-term combination therapy of Pentoxifylline and Tocopherol was established, followed by a sequestrectomy with Advance Platelet Rich Fibrin membranes application. After 16 months of treatment and periodic check-ups, symptoms resolved completely with complete healing of the orocutaneous fistula and preservation of the alveolar and mandibular bones. Multiple guidelines have been proposed to treat osteoradionecrosis. However, the vast majority are based on radical treatments that generate physical and psychological consequences for patients. Therefore, conservative surgical management combined with PENTO and A-PRF could play a role in managing advancedstage ORN in cases that showcase contraindications for mandibular microvascular reconstruction.
\end{abstract}

\section{Keywords}

Osteoradionecrosis, Pentoxifylline, Alpha-Tocopherol, A-PRF, Mouth neoplasms

\section{Introduction}

Osteoradionecrosis (ORN) of jaws corresponds to one of the most severe and debilitating radiation therapy complications for treating malignant head and neck pathologies [1]. Currently, ORN is defined as the exposure of necrotic bone that persists for more than three months in a previously irradiated area at a dose greater than $50 \mathrm{~Gy}$ and that is not caused by recurrence of a tumor [2]. The reported incidence of ORN ranges from 2.6 to $15 \%$, and it occurs between 22 and 47 months after radiation treatment [3].

Regarding the pathophysiology of ORN, Delanian, et al. [4] postulated that ionizing radiation induces a fibroatrophic process, which through tissue damage and the consequent release of reactive oxygen species, generates a non-regulation of fibroblast activity.

Among the risk factors associated with ORN development are the location of the primary tumor, the stage of cancer (III, IV), acritical radiation dose $>50 \mathrm{~Gy}$, previous surgery on the primary tumor

Citation: Valladares PS, Gahona GO, Romero AP, Vargas BJP (2021) Pentoxifylline-Tocopherol and A-PRF Associated with Sequestrectomy for the Treatment of Severe Mandibular Osteoradionecrosis: Case Report and Long-Term Follow-Up. Res Rep Oral Maxillofac Surg 5:057. doi.org/10.23937/26433907/1710057

Accepted: August 28, 2020; Published: August 30, 2021

Copyright: (C) 2021 Valladares PS, et al. This is an open-access article distributed under the terms of the Creative Commons Attribution License, which permits unrestricted use, distribution, and reproduction in any medium, provided the original author and source are credited. 
(mandibulectomies or osteotomies before radiation), poor oral hygiene, dental extractions or bone trauma after radiotherapy (RT), tobacco and alcohol consumption, and diabetes mellitus [3]. On the other hand, different ORN classifications exist. The most accepted classification corresponds to the stages of Notani, et al. [5], who through the clinical and radiographic description of the mandibular bone, divided the ORN into three stages (I, II, III) ascending in gravity.

ORN management is controversial. Small areas of exposed bone have traditionally been treated by conservative management consisting of mouthwashes, analgesia, and antibiotic therapy for acute infections. Local debridement and sequestrectomy have been suggested for grade II ORN, while for advanced ORN stages, such as grade III, wide resection with immediate microvascular reconstruction has been used [1,4,5].

This report aims to present the clinical management of a long-standing ORN patient with a posterior pathological fracture due to floor of mouth cancer based on Pentoxifylline- Tocopherol (PENTO), Advanced Platelet Rich Fibrin (A-PRF) and conservative surgical treatment

\section{Case Report}

A 61-year-old male patient diagnosed with ORN with one year of development posterior to the extraction of tooth 3.6, which was treated at another medical center, consulted to our Oral and Maxillofacial surgery department.

Within the morbid antecedents, a history of a squamous cell carcinoma on the mouth floor (T1, NO) was diagnosed in 2010, treated by local removal surgery and post-surgical radiation with a total radiation dose of $60 \mathrm{~Gy}$. Also, the patient has insulin-requiring type II diabetes mellitus (IRDM), severe arterial hypertension, heart failure, rheumatoid arthritis, bronchial asthma, and a history of acute myocardial infarction. Among the drugs consumed were NPH insulin (am/pm), metformin, acetylsalicylic acid, losartan, hydrochlorothiazide, and salbutamol. Concerning habits, tobacco consumption for more than 30 years, which was not suspended after oral cancer treatment.

The extraoral examination revealed a volume increase and a cutaneous fistula with purulent drainage in the left submandibular area. On intraoral examination, an upper partial edentulism with multiple cervical caries, and a mandibular edentulism with an increase in volume in the left alveolar ridge, with bone exposure in the tooth area 3.6. Also, a $3 \mathrm{~mm}$ mobile bone sequestration was observed in the lower lingual area, and a vestibular bone exposure of $2 \mathrm{~mm}$. The patient was hospitalized for decompensated IRDM and intravenous antibiotic therapy with Ceftriaxone $2 \mathrm{gr} /$ day and Clindamycin 600 mg every 8 hours. An Orthopantomography (OPG) was requested, which revealed diffuse radiolucent areas compatible with pathological mandibular fracture and basilar bone sequestration (Figure 1). A computerized axial tomography (CT) revealed diffuse hypo and hyperdense areas with destruction processes and multiple bone sequestration that reached the basilar border. Besides confirming ORN diagnosis and ruling out tumor recurrence at the affected site, a positron emission tomography (PET-CT) was requested, which showed isolated bone fragments compatible with sequestration and bone metabolic hyperactivity suggestive of osteomyelitis, confirming the diagnosis of ORN Notani stage III (Supplementary Figure 1B, Supplementary Figure $1 \mathrm{C}$ and Supplementary Figure 1D). An angio-CT of inferior extremities was requested to evaluate the eventual mandibular reconstruction with fibula free flap. The images showed advanced multiple atheromatosis, which within the context of the multiple comorbidities of the patient contraindicated this procedure.

After stabilizing IRDM and a favorable response to antibiotic therapy with decreased inflammatory

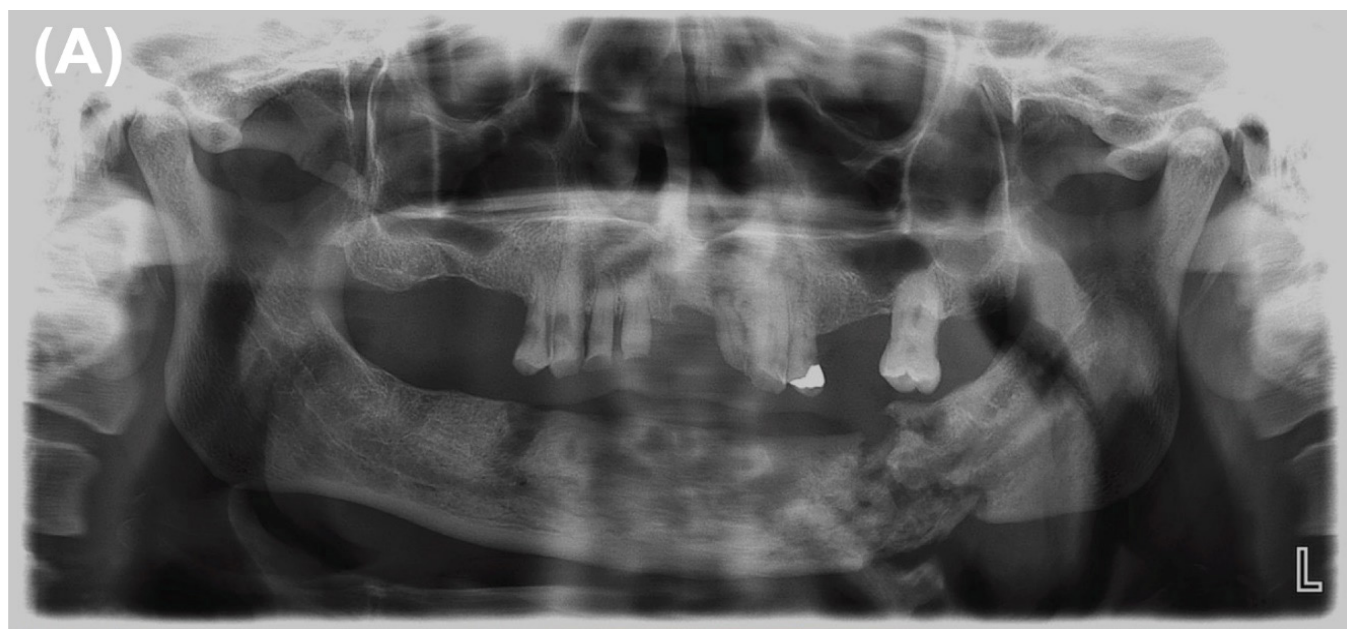

Figure 1: Diagnostic OPG where a fracture in the pathological bone and the presence of multiple bone sequestration are observed. 
parameters and local clinical improvement, hospital discharge was granted. Conservative treatment was started with Amoxicillin $875 \mathrm{mg}+$ Clavulanic Acid $125 \mathrm{mg}$ every 12 hours $\times 14$ days, plus mouthwashes with $0.12 \%$ chlorhexidine and fistular irrigation. A pap-like regimen, smoking cessation, and optimization of hyperglycemia management were indicated.

After the initial treatment, PENTO therapy (Pentoxifylline $400 \mathrm{mg}$ and Tocopherol $400 \mathrm{IU}$, both every 12 hours) was established on a permanent basis. It was planned that three months after starting PENTO, a basilar fragment sequestrectomy, surgical cleaning, and eventual mandibular stabilization with external devices would be performed; however, due to the COVID-19 pandemic, it could not be performed on the estimated time. Thereby, PENTO was kept for approximately ten months. During this period, clinical improvement was observed on the oral mucosa level without areas of bone communication and minimum purulent secretion.

The remaining maxillary teeth extractions were performed in two surgical stages using atraumatic technique and socket filling with Advanced Platelet Rich Fibrin (A-PRF) plugs due to decreased local trauma in the affected area. Once elective surgical procedures were allowed during the pandemic period, we started with injections of liquid fibrin-rich plasma before lifting the intraoral mucoperiosteal flap to expose the affected site on the left mandible. Then, a surgical cleaning was performed to remove basilar bone sequestration, necrotic tissue, washing with injectable Clindamycin directly into the affected site, filling with A-PRF membranes [6] and fistulectomy. Notably, during intraoral exposure of the mandibular alveolar ridge, the presence of a fibrous callus was observed on the affected area without mobility in the margins, for which it was decided intraoperatively not to stabilize the bone fragments (Figure 2).

A favorable clinical evolution was observed in the post-surgical controls with the oral mucosa's healing (Supplementary Figure 2). Also, there was an absence of inflammatory-infectious signs, and complete remission of the cutaneous fistula was seen (Supplementary Figure 3). As of this report's date, the patient remains with PENTO therapy, completing a total period of 16 months with monthly clinical and radiographic controls. Radiographically, initial signs of ossification in the alveolar area of the compromised region, and an absence of osteolysis and of new bone sequestration have been observed (Supplementary Figure 4).

\section{Discussion}

This case presented a favorable response to conservative management of a Notani III stage ORN, where surgical resection and subsequent microvascular graft are the conventional choice therapies. To our knowledge, this report is one of the few that combined conservative surgical management and drug therapy in a patient with multiple comorbidities [1-3,5].

When radical surgery with microsurgical reconstruction is not possible, conservative pharmacological therapy has been proposed on the basis of the pathophysiological mechanisms of ORN with a long-term regimen $[1,4,7]$. The use of PENTO has been proposed in order to counteract the effects of ORN-associated fibroatrophy phenomena. Pentoxifylline corresponds to a drug that has been shown to have vasodilator, TNF-a suppressing, inhibition of inflammatory reactions and platelet aggregation, along with an increase in erythrocyte flexibility [4-7]. Moreover, Tocopherol is a potent antioxidant effective in inhibiting free radicals. The use of PENTO has been reported, in patients with advanced ORN, not suitable for resection due to comorbidities. Delanian, et al. [4] described the treatment of 6 patients with pathological fractures, where 5 showed clinical improvement with resolution of the fracture. Breik, et al. [7] reported two patients with pathological fractures and orocutaneous fistulas successfully treated with PENTO. A systematic review that included patients with advanced-stage ORN or refractory to other treatments suggested considering the administration of PENTO for a minimum of 6 months and until regression of the condition is ob-

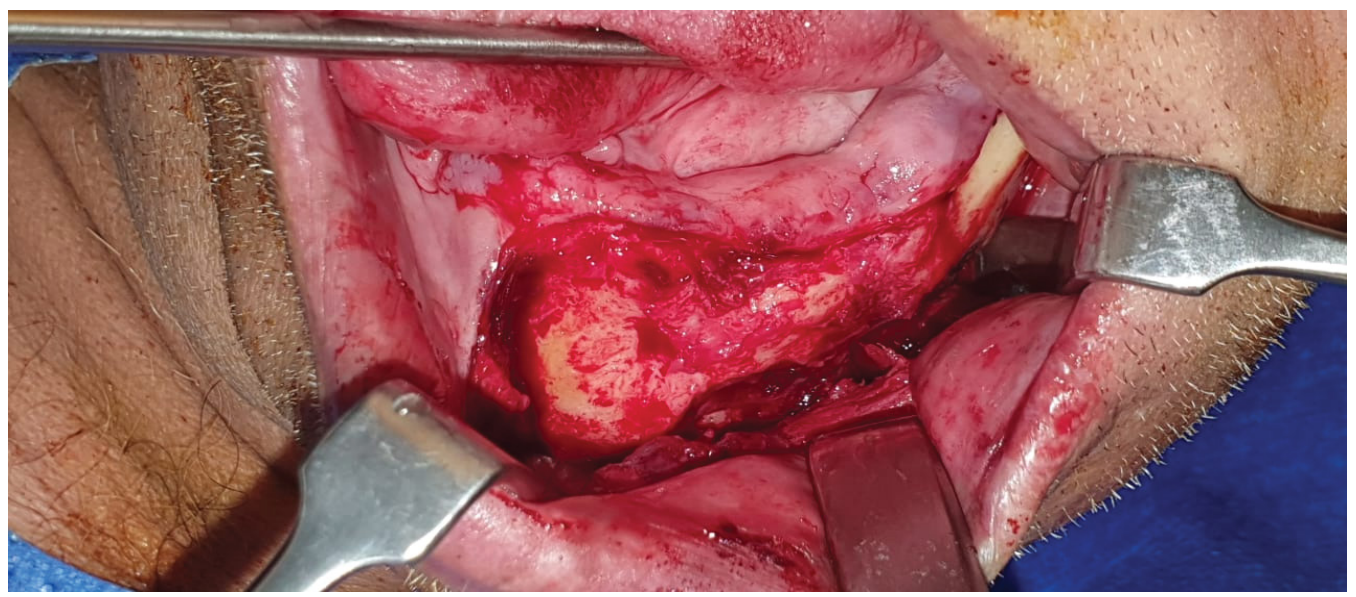

Figure 2: Intraoperative view showing fibrous callus formation in a site previously affected by ORN. 
served. Also, a proportion estimate of $60 \%$ recovery was obtained in patients treated on a PENTO basis [8].

Another complementary technique is the use of A-PRF [6]. This fibrin network obtained from blood seeks to accumulate platelets, cytokines, and growth factors to optimize local angiogenesis, an essential process to achieve adequate tissue restoration. Other studies have shown that A-PRF acts as a reservoir of bioactive molecules that promote tissue repair and bone regeneration, induced by cell proliferation, migration, and differentiation in conjunction with anti-inflammatory properties. It is imperative that the affected area is free of malignant neoplasia and that there is no metastasis within the area where A-PRF will be applied, since the growth factors contained therein also play a key role in tumor angiogenesis [9].

Reasonable control of oral hygiene translates into a $1 / 3$ reduction in ORN appearances. Otherwise, patients with a history of addictions show an increase in the incidence of ORN. Diabetes mellitus is considered the most determining systemic factor for the appearance of ORN (Hazard Ratio: 2.99), being also the most frequent systemic pathological condition among these patients, considering that such population is more susceptible to development of infections $[2,10]$.

Finally, conservative surgical management combined with PENTO and A-PRF could play a role in advanced-stage ORN development in cases in which a contraindication for microvascular mandibular reconstruction exists. In the case presented, the patient showed a union of the pathological fracture, remission of the orocutaneous fistula, and healing signs. Although randomized clinical trials are lacking, this management could be a minimally invasive surgical treatment.

\section{Acknowledgment}

None.

\section{Competing Interest}

The authors report no competing interests.

\section{Ethical Approval}

An exemption statement was obtained from the ethics board.

\section{Patient Consent}

Written consent was obtained for publication.

\section{Funding}

No funding was received for this study.

\section{References}

1. Rice N, Polyzois I, Ekanayake K, Omer O, Stassen LF (2015) The management of osteoradionecrosis of the jaws-a review. Surgeon 13: 101-109.

2. McCaul JA (2014) Pharmacologic modalities in the treatment of osteoradionecrosis of the jaw. Oral Maxillofac Surg Clin North Am 26: 247-252.

3. O'Dell K, Sinha U (2011) Osteoradionecrosis. Oral Maxillofac Surg Clin North Am 23: 455-464.

4. Delanian S, Chatel C, Porcher R, Depondt J, Lefaix JL (2011) Complete restoration of refractory mandibular osteoradionecrosis by prolonged treatment with a pentoxifylline-tocopherol-clodronate combination (PENTOCLO): A phase II trial. Int J Radiat Oncol Biol Phys 80: 832-839.

5. Notani K, Yamazaki Y, Kitada H, Sakakibara N, Fukuda H, et al. (2003) Management of mandibular osteoradionecrosis corresponding to the severity of osteoradionecrosis and the method of radiotherapy. Head Neck 25: 181-186.

6. Choukroun J, Diss A, Simonpieri A, Girard MO, Schoeffler C, et al. (2006) Platelet-rich fibrin (PRF): A second-generation platelet concentrate. Part V: histologic evaluations of PRF effects on bone allograft maturation in sinus lift. Oral Surg Oral Med Oral Pathol Oral Radiol Endod 101: 299-303.

7. Breik O, Tocaciu S, Briggs K, Tasfia Saief S, Richardson S (2019) Is there a role for pentoxifylline and tocopherol in the management of advanced osteoradionecrosis of the jaws with pathological fractures? Case reports and review of the literature. Int J Oral Maxillofac Surg 48: 1022-1027.

8. Kolokythas A, Rasmussen JT, Reardon J, Feng C (2019) Management of osteoradionecrosis of the jaws with pentoxifylline-tocopherol: A systematic review of the literature and meta-analysis. Int J Oral Maxillofac Surg 48: 173-180.

9. Strauss FJ, Nasirzade J, Kargarpoor Z, Stähli A, Gruber R (2020) Effect of platelet-rich fibrin on cell proliferation, migration, differentiation, inflammation, and osteoclastogenesis: A systematic review of in vitro studies. Clin Oral Investig 24: 569-584.

10. Dumoulin S, van Maanen A, Magremanne M (2020) Dental prevention of maxillo-mandibular osteoradionecrosis: A ten-year retrospective study. J Stomatol Oral Maxillofac Surg 122: 127-134. 


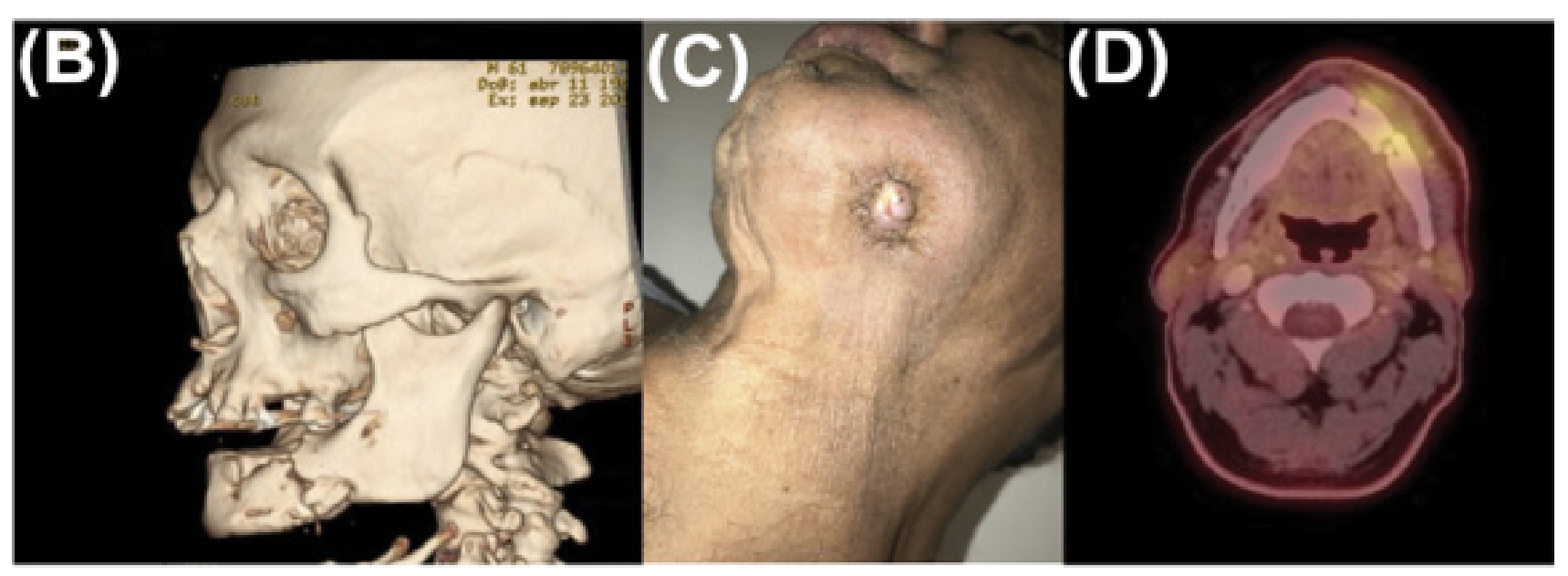

Supplementary Figure 1: B) CT-Scan showing a pathological fracture in the left mandibular body, with the presence of multiple bone sequestration; C) Active orocutaneous fistula in the skin of the left submandibular region associated with refractory ORN and D) PET-CT with areas of mandibular bone metabolic hyperactivity.

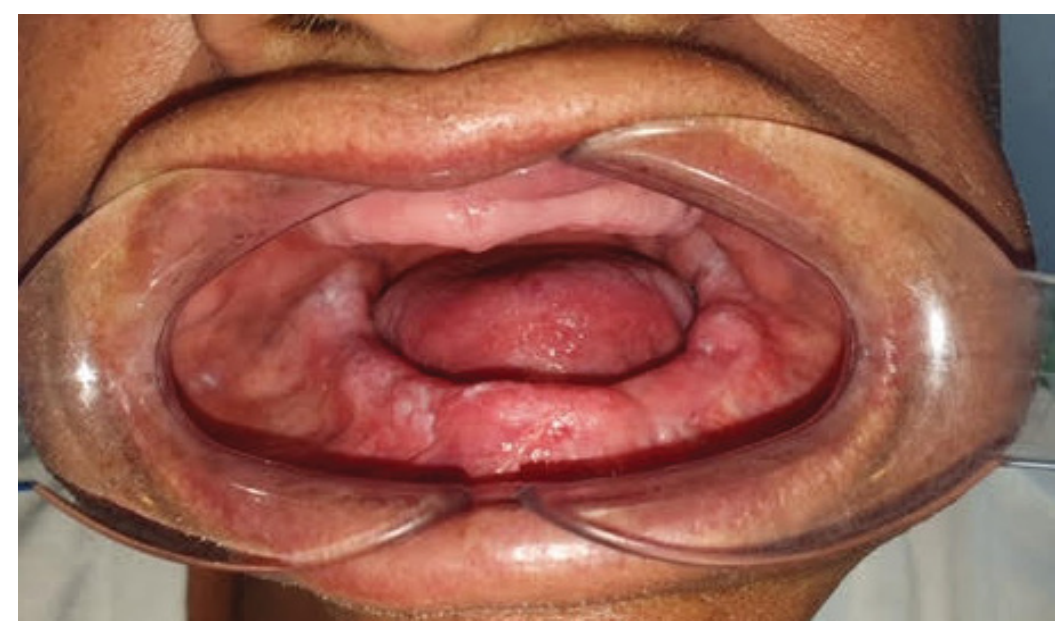

Supplementary Figure 2: Post-operative control 6 months after the sequestrectomy plus the application of A-PRF membranes, which shows a healthy mucosa without signs of inflammation and suppuration.

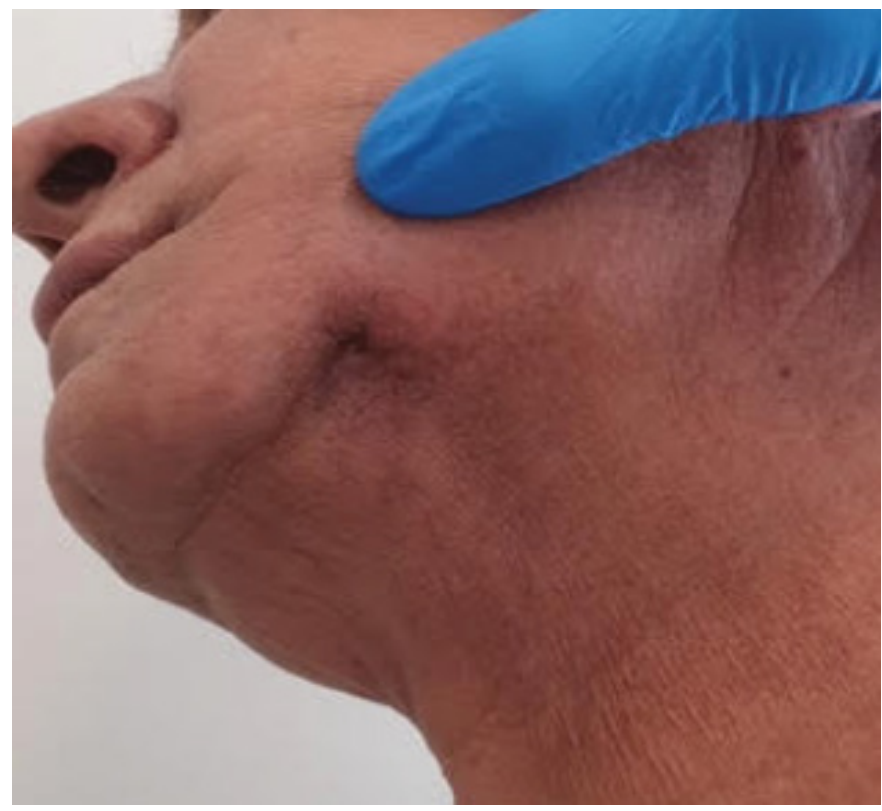

Supplementary Figure 3: Lower view of the jaw showing complete healing of the orocutaneous fistula. 


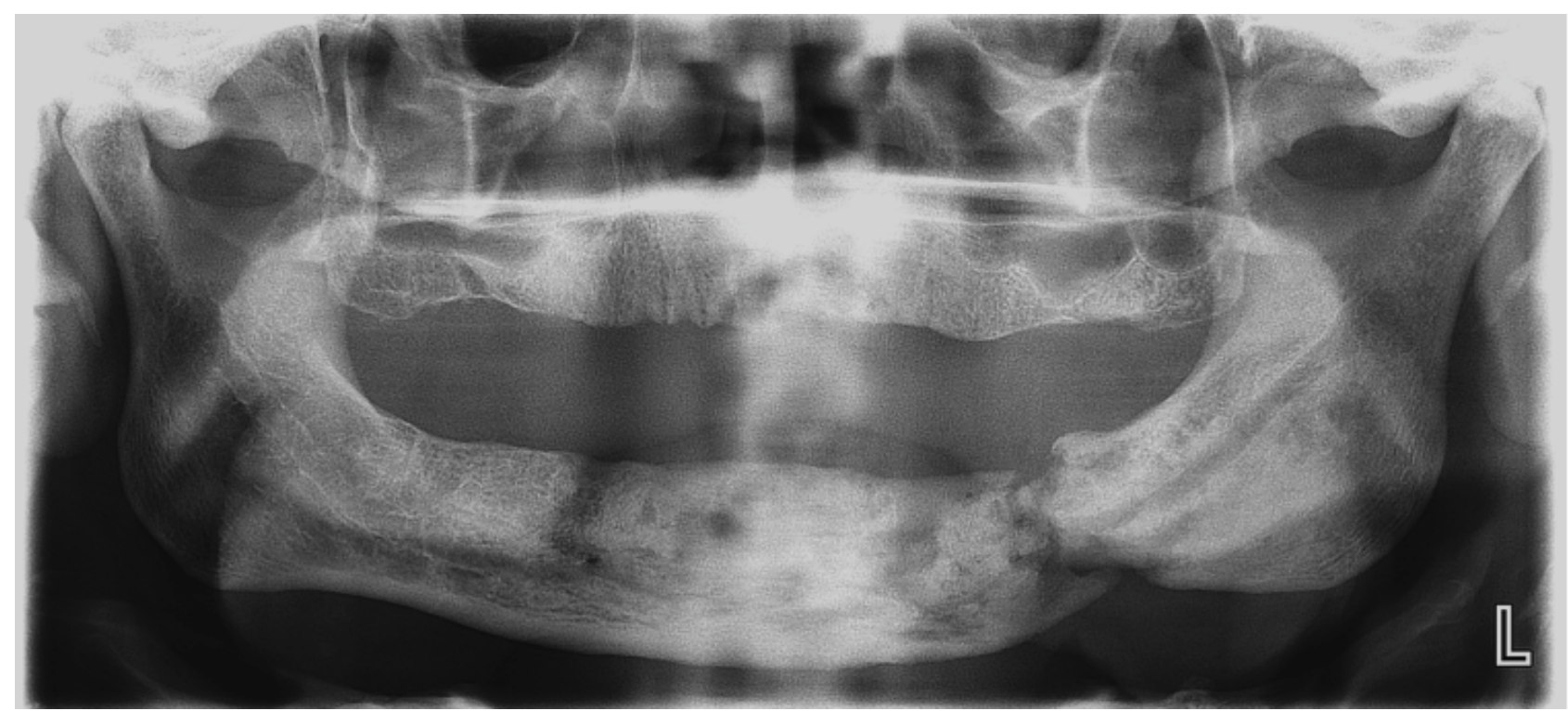

Supplementary Figure 4: OPG control at 16 months after PENTO. Radiopaque areas are observed between fractured strands suggestive of initial ossification stages. 